\title{
Skin-Sparing Mastectomy: An Update for Clinical Practice*
}

\author{
Kelly Lambert $^{1,2}$, Kefah Mokbel ${ }^{1 \#}$ \\ ${ }^{1}$ The London Breast Institute, The Princess Grace Hospital, London, UK \\ ${ }^{2}$ The Breast Unit Glenfield Hospital, University Hospitals Leicester, Leicester, UK \\ Email: "kefahmokbel@hotmail.com
}

Received September 9, 2012; revised October 10, 2012; accepted October 20, 2012

\begin{abstract}
Aim: To review the oncological safety and aesthetic advantage of skin-sparing mastectomy (SSM) for invasive breast cancer (IBC) and ductal carcinoma in-situ (DCIS). Controversies including the impact of radiotherapy (RT) on immediate breast reconstruction (IBR), preservation of the nipple-areola complex (NAC) and the role of endoscopic mastectomy are also considered. Methods: Literature review using Medline and PubMed. Results: SSM is safe in selected cases; including IBC $<5 \mathrm{~cm}$, multi-centric tumours, DCIS and for risk-reduction surgery. Inflammatory breast cancers and tumours with extensive involvement of the skin represent contra-indications to SSM due to an unacceptable risk of local recurrence. SSM can facilitate IBR and is associated with an excellent aesthetic result. Prior breast irradiation or the need for post-mastectomy radiotherapy (PMRT) do not preclude SSM, however the cosmetic outcome may be adversely affected. Nipple/areola preservation is safe for peripherally located node negative tumours. A frozen section protocol for the retro-areolar tissue should be considered in these cases. The advent of acellular tissue matrix systems has widened the applicability of implant-based immediate reconstruction following SSM. Data on endoscopic mastectomy is limited and superiority over conventional SSM has not been demonstrated. Conclusion: SSM is safe in selected cases and is associated with advantages over simple mastectomy, including a superior aesthetic outcome and a potential reduction in the number of reconstructive procedures per patient.
\end{abstract}

Keywords: Natural Asset; Financial Value; Neural Network

\section{Introduction}

Breast-conserving surgery combined with adjuvant radiotherapy is safe as an alternative to mastectomy for the majority of women with early invasive breast cancer (IBC) [1]. However, up to one third of patients require a mastectomy for large or multi-focal tumours (particularly where breast conservation would lead to a poor cosmetic outcome), local recurrence and patient preference [2].

The primary aim of surgery to the breast and axilla in breast cancer is to achieve local control of disease and to provide prognostic information to plan adjuvants. A good aesthetic outcome is also desirable. For patients who have undergone mastectomy, a breast reconstruction can help achieve this but the optimum timing remains controversial with guidance from level-1 evidence lacking. Immediate breast reconstruction (IBR) can result in a poorly contoured breast, with prominent scars and a paddle of skin that is of a different colour and texture [3]. Delayed reconstruction mandates at least a second surgical procedure.

\footnotetext{
"Conflicts of interest: none declared.
}

\#Corresponding author.
Skin-sparing mastectomy (SSM), a type of mastectomy where incisions are planned to maximise skin preservation and facilitate breast reconstruction, was described by Toth and Lappert in 1991 [4]. SSM involves en-bloc removal of the breast gland, nipple-areola complex (NAC), previous biopsy sites and the skin overlying superficial tumours. The native breast skin envelope and inframammary fold are preserved [5]. Using the native skin envelope optimises the final contour of the reconstructed breast reducing the need for contralateral breast adjustment in order to achieve symmetry. Scarring and donor skin requirements (in flap based reconstructions) are minimised [6]. A combined oncological and reconstructive approach is acceptable to patients, cost-effective and reduces the number of hospital admissions and time away from home or work [7]. This approach has been advocated as an effective treatment option for patients with early-stage IBC and DCIS which is not amenable to breast conserving therapy. SSM and IBR has also been shown to be of particular use in patients who develop breast cancer following augmentation mammoplasty who might otherwise have a poor cosmetic result with breast 
conserving surgery and adjuvant radiotherapy. SSM and IBR can allow the omission of adjuvant RT and is associated with an excellent cosmetic outcome [8].

Acceptance and popularity are increasing amongst patients and many surgeons. A 2008 survey of Californian surgeons performing surgery for breast-cancer seems to confirm a change in attitudes towards SSM, with $90 \%$ of those surveyed satisfied with the oncological adequacy of the technique in early breast-cancer and $70 \%$ in agreement that the cosmetic results of SSM are superior to standard mastectomy [9].

The evidence on oncological adequacy of SSM will be presented in this article. In addition to survival and the risk of LR, post-operative morbidity, local control, cosmesis, patient satisfaction and an assessment of functional disturbance and psychological morbidity are important outcome measures [10].

Controversies including the impact of radiotherapy (RT) on IBR, preservation of the nipple-areola complex (NAC) and the role of endoscopic mastectomy are considered. The use of biological grafts (such as Alloderm and Strattice) in immediate reconstruction using subpectoral implants is also discussed.

\section{Surgical Considerations}

The management of women with IBC should take place within the context of a multidisciplinary team. Techniques such as SSM and IBR are demanding and should be undertaken by breast surgeons with oncoplastic training or as a joint procedure between a general and plastic surgeon. Oncological principles should not be compromised. The incisions used to perform SSM will be influenced by various factors including breast size and ptosis, NAC size, tumour location, position of biopsy sites, need for axillary intervention, choice of reconstructive technique and the preference of the surgeon. Many patients are suitable for a peri-areolar approach (Figure 1) where the only breast skin excised is the NAC $[5,11]$. If the NAC is small and the breast is large, a peri-areolar incision with lateral extension or a larger ellipse including

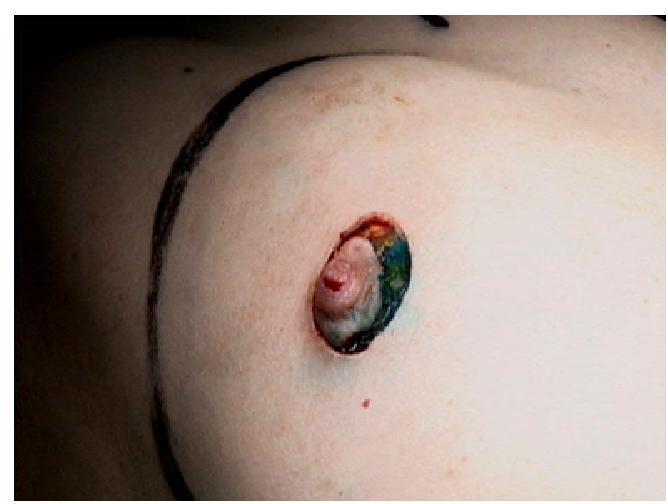

Figure 1. The incision of standard SSM. the NAC can be used. Wise-pattern incisions, akin to those used for reduction mammoplasty, can be required for large breasts with moderate/severe ptosis [12]. If necessary, symmetry may be achieved by performing a simultaneous, or delayed, contralateral reduction mammoplasty.

Some surgeons advocate the subcutaneous injection of saline-adrenaline solution $(1: 25,000)$ when performing SSM. This is haemostatic and results in some hydrodissection of the plane between the subcutaneous tissue and the mammary gland. Skin flaps are carefully elevated and the dissection is extended to the edges of the breast tissue circumferentially, preserving the infra-mammary fold [13]. High intensity fibre-optic light sources and longer instruments can be useful during this stage as access is limited due to the smaller incisions. The parenchyma can then be mobilised by dissecting the pectoral fascia from the underlying musculature. In order to protect the viability of the preserved envelope, skin flaps must be sufficiently thick to maintain the sub-dermal vascular plexus without leaving breast tissue behind. Care is required to limit excessive use of electro-cautery and flap margins should be assessed for bleeding. Native skin flap necrosis (partial or complete) has been estimated to occur in $11 \%$ of cases which is similar to conventional mastectomy [14].

Sentinel lymph node biopsy (SLNB) and/or axillary node clearance (ANC) can be performed safely through the same incision $[6,11,15]$. In larger breasts an additional axillary incision will sometimes be required [3]. SLNB should be performed in patients with IBC and a clinically negative axilla. Some surgeons perform intra-operative assessment of the sentinel node (frozen section, imprint cytology or one step nucleic acid amplification-OSNA) and perform ANC prior to IBR if the SNLB is positive. However, intra-operative analysis can be associated with false negatives and ANC is more difficult to perform as a second operation following IBR, with potential to harm to the vascular pedicle and viability of a flap-based reconstructed breast [16,17]. An alternative approach is to perform a day-case sentinel node biopsy one or two weeks before the mastectomy so that nodal status is known [18].

Endoscopic video-assisted surgery has been successfully employed for a range of aesthetic and plastic surgery procedures including those relating to the breast. The technique has been extended to the treatment of both benign and malignant breast conditions [19]. Yamashita et al. have described the use of a $2.5 \mathrm{~cm}$ axillary incision and skin traction to create a working space, allowing partial or total glandular resection and SLNB $+/-$ ANC to be successfully performed. Operating times and blood loss were comparable to open surgery and all surgical margins were clear. The conversion rate to open surgery 
was low. Wounds healed with minimal scarring and the approach was associated with high levels of patient satisfaction [20]. Similarly, small peri-areolar incisions can be made to facilitate endoscopic breast conserving surgery for early-stage IBC $[21,22]$. Some studies have also reported immediate reconstruction with the latissimus dorsi (LD) flap [23] or prosthetic implant [24]. Kitamura et al. compared patients undergoing SSM and IBR, using an implant, with or without endoscopic assistance. The addition of endoscopy was associated with a longer operating time but significantly smaller scars and greater patient satisfaction [25]. It is noteworthy that these reports are from relatively small studies from single institutions and superiority over conventional techniques has not been established.

\section{Reconstructive Options Following SSM}

Choice of reconstructive technique following SSM requires careful consideration of several patient related factors, including: breast size, ptosis, areola size, patient preference and expectation, general health and smoking status. Tumour related factors include size, location and proximity to the NAC. Preference and experience of the operating surgeon is also taken into account. Occasionally patients may refuse reconstruction or the prognosis may be so poor that any breast reconstruction is unwarranted [26]. In all other cases, the breast can be reconstructed with prosthetic implants (Figure 2) and/or autologous tissue (Figure 3). Following SSM, myocutaneous flaps allow replacement of the small area of excised skin and aim to approximate the complete volume of breast tissue removed with or without the assistance of a prosthetic implant. Single-stage reconstruction of the breast mound can then be followed by techniques to address the nipple and areola [27].

Conventional mastectomy is associated with significant excision of the native skin envelope, complicating implant-only reconstruction. It is most often necessary to use an expandable implant and to exchange this at a sec-



Figure 2. Bilateral SSM and immediate implant based reconstruction with subsequent nipple reconstruction. Patient also had adjuvant radiotherapy to the right breast.

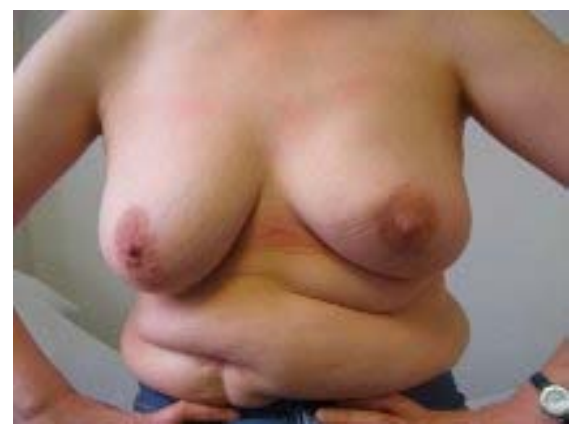

Figure 3. Patient is a 50 years old lady who underwent left SSM and immediate breast reconstruction with LD flap and implant. Nipple reconstructed subsequently.

ond operation once the remaining skin has stretched to accommodate the desired volume over multiple out-patient inflations. Although expandable implants are stillcommonly used, the advent of SSM and anatomically profiled implants has meant that implant-based reconstruction can now often be performed in a single stage procedure, or if a two stage procedure is needed, the number of inflations can be minimised. The implant is positioned in a sub-muscular pocket superiorly and ideally a sub-fascial pocket inferiorly, allowing full coverage of the prosthesis. The results of this approach in terms of breast volume, shape and symmetry are favourable with complication rates as low as $8.3 \%$ [28]. A prospective study of 400 implant only reconstructions following SSM, identified patient related risk factors associated with complications. The authors recommend particular caution in obese patients who smoke (32\% loss of implant) and in large breasted women (27\% loss of implant) [29]. In a separate study, the same author found patient age and experience of the operating surgeon to be associated with a greater risk of post-operative complications [30].

Tissue matrix (TM) products such as AlloDerm ${ }^{\circledR}$, SurgiMend $^{(\mathbb{R}}$ and Strattice ${ }^{\mathbb{B}}$ have expanded the use of implant-based reconstruction. TM is manufactured from donor human skin $\left(\right.$ AlloDerm ${ }^{\circledR}$ ), bovine dermis (SurgiMend $\left.^{(}\right)$or porcine dermis (Strattice ${ }^{\circledR}$ ) denuded of epidermis and aseptically processed. This results in an intact acellular matrix of natural biological components that promotes rapid revascularization and cell repopulation. TM products have been shown to incorporate well into tissues with no rejection. In implant-based breast reconstruction, they are used to cover the inferior pole of an implant meaning that a larger initial size may be used thus allowing SSM and immediate breast reconstruction using a fixed volume prosthesis as a single stage procedure. A recent study which compared use of TM versus conventional sub-pectoral implant, revealed significantly larger initial fill volumes and fewer sessions required for inflation with no differences in complication rates be- 
tween the two groups [31]. A non-randomised large series of 361 women undergoing implant with or without TM did not reveal any differences in infection rates between two groups [32]. However, TM was associated with adherence to skin in all cases, particularly after RT.

The LD flap, introduced by Schneider in 1977, was once the standard for autologous breast reconstruction [33]. A variety of other flaps and prosthetic materials are now available but the LD myocutaneous flap is still in common usage. The LD is often combined with an implant for use in a single-stage procedure, where it serves to improve coverage of the prosthesis and improve the final breast contour [34].

The TRAM (Transverse Rectus Abdominus Myocutaneous flap) [35] can be per formed either as a local pedicle or a free tissue transfer flap [36]. In one study, comparing 85 patients undergoing TRAMs, implant-based or LD reconstructions the mobility and consistency of the reconstructed breast more closely resembled the natural breast in TRAM flap patients [37]. Patients also report high levels of satisfaction with the aesthetic result [38]. However, TRAM flap reconstruction can be associated with donor site complications.

The DIEP flap, described by Holmstrom [39] and popularised by Blondeel [40], allows large volume tissue transfer with donor site advantages over the TRAM flap. Preservation of total abdominal musculature and aponeurotic layers reduces complications such as muscular weakness, asymmetry, bulging and hernias [41]. The DIEP and superficial inferior epigastric artery (SIEA) flaps are able to transfer the same tissue from the abdomen as the TRAM flap without sacrificing the rectus muscle or fascia [27]. Furthermore, the neo-breast consists primarily of fat and skin like the native organ and patients benefit from an improved abdominal contour. However, the procedure can be technically demanding, in particular the limited surgical access associated with SSM can cause difficulties in the identification and dissection of recipient vessels.

In a series of 30 DIEP flap breast reconstructions, with an average follow-up of 29 months, breast skin complications were noted in 2 patients manifesting as small areas of necrosis. Partial losses were observed in 2 patients representing less than $15 \%$ of the total area. There was only one case of total flap loss and one case of local recurrence during the follow-up period [42]. Patients reported high levels of satisfaction with the outcome. In a study of 42 patients, SSM and IBR using the DIEP flap was compared to breast conservation and found to be associated with comparable quality of life and significantly better cosmetic outcome [43].

The use of soft tissue from the gluteal region as a free flap was introduced by Fujino et al. in 1975 [44]. In 1995, the free superior gluteal artery perforator (S-GAP) flap was used for autologous breast reconstruction [45]. This allowed free transfer of skin and fat without sacrifice of the gluteus maximus, thereby minimising donor site complications. In addition it is sometimes possible to anastomose nerves, harvested with the flap, to local branches at the recipient site and provide limited sensation to the flap [40]. This approach is particularly useful when the abdomen is not suitable or preferable as a donor site. In a study of 142 GAP flaps, the superior-GAP flap was found to have significant advantages over the inferior-GAP flap. The authors reported low morbidity, $98 \%$ flap survival, good cosmetic results and high levels of patient satisfaction [46].

Several other free flaps have been described for autologous breast reconstruction including the myocutaneous transverse upper gracilis free flap (TUG) $[47,48]$. There are also reports in the literature of free omental flaps, harvested laparoscopically, being used for IBR following SSM [49].

In a survey of 453 female plastic surgeons from USA and Canada questioned on which method of reconstruction they would choose if they had breast cancer, $66 \%$ preferred implant based over autologous breast reconstruction [31].

\section{Oncological Considerations}

The main oncological concern in SSM is that residual breast tissue within the skin envelope may manifest later as LR. Histological studies following conventional mastectomy have confirmed the presence of residual glandular tissue in $5 \%$ of all biopsies taken from the operating field [50]. Torresan et al. performed histological analysis of skin sparing mastectomy flaps from patients with IBC who were marked up for SSM and then had conventional mastectomy performed and found the prevalence of residual breast tissue to be $59.5 \%$ and residual disease to be $9.5 \%$ in the portion of the specimen that would have been left in-situ had the patient undergone SSM. The presence of breast tissue and residual disease was significantly associated with skin flaps thicker than $5 \mathrm{~mm}$ [51]. Ho et al. performed histological examinations of the skin and subcutaneous tissue of 30 conventional mastectomy specimens and found that the skin flaps (excluding the NAC) were involved in $23 \%$ ( 7 of 30 ) of cases. In 5 cases, the skin involved was situated directly over the tumour. Significant risk factors for this were skin tethering, large tumour size and peri-neural infiltration [52]. Despite this, the incidence of LR following SSM for IBC has been investigated by several authors and found to comparable to conventional mastectomy [53].

Although there are no large randomised trials comparing conventional to SSM, a meta-analysis of 9 observational studies has been published. In this study, 3739 patients were included, $30 \%$ of which underwent SSM. 
The median follow-up in the included studies was between 15 and 101 months [54]. All the included studies were retrospective and non-randomised, but there was no significant difference in stage or grade between the 2 groups. This meta-analysis found no significant difference in local recurrence between SSM and standard mastectomy (SSM $3.8 \%-10.4 \%$ vs $1.7 \%$ - 11.5\%). The B06 trial (a randomized clinical trial comparing total mastectomy and segmental mastectomy with or without radiation in the treatment of breast cancer) found a roughly equivalent $10 \%$ local recurrence rate at 20 years in the mastectomy treated group [1].

A large retrospective series from a single centre recently reported outcomes in 1810 patients undergoing either SSM (799 patients) or standard mastectomy (1011 patients). After adjusting for disease stage and age there was no significant difference in disease-free survival or local recurrence rates [55].

Many patients requiring mastectomy have T3 tumours. Though evidence for the safety of SSM for these tumours is less clear, the results of early studies are encouraging. In a study of 38 patients with tumours considered to be at high risk of LR, only one case $(2.6 \%)$ developed a LR after SSM and IBR after a median 53 months of followup, despite 10 (26\%) systemic recurrences [56]. Foster et al. performed SSM and IBR on a prospective cohort of 25 patients with stage $2 \mathrm{~b}$ or 3 disease and reported an overall post-operative complication rate of $13 \%$ and only one LR after 4 years of follow-up [57].

Neo-adjuvant chemotherapy may be administered to T3 tumours in an attempt to down-stage disease. This may facilitate SSM in a breast which may otherwise have required conventional mastectomy. Alternatively, it may even facilitate breast conservation surgery, avoiding the need for a mastectomy altogether. Radiotherapy should be given to all patients with locally advanced or high risk breast cancers, however its timing in relation to treatments such as SSM and IBR has yet to be established.

It is unlikely that large multi-centre randomised studies comparing conventional mastectomy and SSM will take place. Although the above studies are retrospective and relatively small, there is reasonable evidence that SSM is a safe oncological operation for T1, T2 and multi-centric tumours. Moreover, there is evidence that SSM combined with IBR does not significantly delay adjuvant therapy, as some clinicians had feared [58].

The predictors and risk factors for LR after SSM relate to both tumour and patient characteristics. LR may be a reflection of the underlying tumour biology rather than the amount of skin preserved during SSM or the choice of reconstructive technique. Many of these studies found that tumour size, stage, lymph node positivity and poor differentiation were all risk factors for LR [7,59]. The management of LR after SSM and IBR can be problem- atic and LR is associated with poor prognosis [55]. LR can be treated locally with surgical excision and RT and systemically with chemotherapy or endocrine therapy [53]. Removal of the reconstructed breast/implant may be required. The anatomical location of recurrence has been shown to have prognostic implications. In a study by Langstein et al. cutaneous or subcutaneous LR was associated with a better overall survival and lower risk of distant metastases and better response to treatment than LR within the chest wall [60].

\section{SSM for DCIS}

A mastectomy may be necessary for extensive, multifocal or recurrent DCIS. Some patients with DCIS also request a mastectomy.

The incidence of LR following conventional mastectomy for DCIS ranges from 1\% - 3\% [61], with cure rates of approximately $98 \%$ (breast cancer-specific mortality of $0.59 \%$ ) [62]. SSM and IBR is a good option for women undergoing mastectomy and for DCIS as PMRT (which may adversely affect the appearance of a reconstructed breast) is not usually required [63].

In one series of 95 patients undergoing SSM and IBR for DCIS, 93 (98\%) were alive and disease free after a median follow-up of 3.7 years [64]. The overall local recurrence rate was 3 of $93(3 \%)$. A larger series 175 of patients reported one LR $(0.6 \%)$ after a median of 65 months follow-up [8]. In a retrospective review of 223 patients with DCIS treated by SSM and IBR, the same author reported an LR rate of 3.3\%, with high tumour grade and surgical margins $<1 \mathrm{~mm}$ as risk factors [61] with a mean follow-up of 82 months. Finally, in a retrospective long-term follow-up study of 44 patients who underwent SSM and IBR for DCIS, there were no local or distant recurrences after 9.8 years [65]. These retrospective studies have all demonstrated that SSM and IBR for DCIS is oncologically safe with low recurrence rates. However, prospective randomised data to confirm these findings is not available.

\section{Preservation of the Nipple-Areola Complex}

It has traditionally been held that the NAC should be removed as the NAC and its adjacent ducts may contain tumour cells which have spread distally along the ducts from the primary tumour. This concept was based on older studies that demonstrated occult tumour in the region of the NAC [7]. Recent evidence has shown that the risk of tumour involvement has been overestimated [6668]. In many patients removal of the NAC may be an over-treatment and hence some surgeons have attempted preservation in view of the cosmetic and psychological benefits. 
In a retrospective series of $286 \mathrm{SSM}$ specimens, 16 (5.6\%) were found to contain tumour in the NAC [66]. Nodal positivity, sub-areolar tumour location and multicentricity were significant risk factors. If multi-centric and sub-areolar tumours were excluded, the NAC was involved in $3 \%$ of cases. Another series of 140 mastectomies also found tumour size and nodal positivity to be risk factors for NAC involvement. The primary tumour was situated within $2.5 \mathrm{~cm}$ of the areola in all 22 cases in which the NAC was positive [67]. A retrospective study of 217 mastectomy specimens reported NAC tumour involvement in 23 cases (10.6\%) [68].

Frozen sections of subareolar tissue can be used to attempt to preserve the NAC. In one study of 112 SSMs in women whose breast cancer was more than $2 \mathrm{~cm}$ from the NAC sections were negative for tumour in 61 cases $(54.5 \%)$ enabling NAC preservation. The NAC was excised in the other 51 cases [69]. The cosmetic results after SSM and IBR (using LD or TRAM flaps) were independently evaluated as excellent or good in $91 \%$ $(102 / 112)$ of patients and were significantly better after preservation of the NAC $(\mathrm{P}=0.001)$. Six $(5.4 \%)$ recurrences occurred in 112 patients who underwent SSM compared with $11(8.2 \%)$ of 134 patients who had undergone conventional mastectomy during the same 6year period, although this difference is likely to reflect differences in the cases chosen for NSM. Only one LR occurred in the NAC preservation group [69]. In a retrospective study of 219 mastectomies, $20 \%$ of NACs were found to be involved in total, consisting of $9.4 \%$ of stage $1-2$ tumours and $30 \%$ of stage 3 tumours. The NAC was involved in only $2.5 \%$ of peripheral tumours vs $68 \%$ of central lesions [70]. Tumour size and distance to the nipple on mammography have been identified as independent predictors of NAC involvement which may aid preoperative planning [71]. Axillary lymph node involvement and lymphovascular invasion have also been shown to be significant risk factors [72]. In another study of 115 cases undergoing NSM for prophylaxis (75) or for breast cancer (40), the occult nipple involvement rate was only $5.2 \%[73]$.

Some patients will lose part or all of the NAC after NSM due to impaired NAC vascular supply. This has been reported to occur in between $6 \%$ and $33 \%$ of cases [74-76]. In the latter study, $54 \mathrm{SSMs}$ in 44 patients were performed in which the NAC was clinically thought to be disease-free. Nipple core biopsy frozen sections were performed. 6 out of 54 biopsies were positive, necessitating conversion to conventional SSM. Of the remaining 48 NSMs, 3 cases had partial NAC loss. In another series of 54 SSMs of which 34 had NAC preservation [77], the skin loss was higher in the latter group although LR was similar $(8.3 \%$ when NAC excised; $7.1 \%$ when NAC preserved). Nipple sparing mastectomy (NSM) performed through a skin incision on the lateral aspect of the breast, rather than a peri-areolar skin incision, has been shown to reduce the risk of ischaemic/necrotic complications for the nipple $(2.8 \%$ vs $59.7 \%)$. In addition to ischaemic complications, impaired sensitivity of the NAC seems to affect most patients to some extent, though some resolution with time can occur [78].

Therefore, it would appear oncologically safe to perform SSM with NAC preservation for prophylactic cases, for DCIS and for smaller tumours of low stage provided the tumour is not close to the nipple and a frozen section protocol is followed. However, many such tumours may not merit a mastectomy.

Patients must be informed that NAC excision may still be required if residual carcinoma is identified on definitive histology or if significant necrosis occurs and that sensation may be impaired. Patients with clinically apparent involvement of the NAC or adjacent skin, bloody nipple discharge, inflammatory or retro-areolar cancers should not be offered NAC preservation.

Some groups, have reported using radiotherapy to the NAC in addition to employing a frozen-section protocol $[79,80]$. One study of 106 NSM with intra-operative RT in which two thirds were done for invasive disease, reported one LR at a mean follow-up of 13 months. Around $10 \%$ of patients experienced partial NAC necrosis and 5\% total necrosis [79]. In another study of NAC preservation, 10 patients were administered NAC RT post-operatively. Around half of these experienced some loss of nipple sensation or non significant necrosis [80]. A randomised study with long term follow-up would be required to determine if these approaches improve local control.

An alternative to NAC-sparing SSM is to remove the nipple but preserve the areola-areola-sparing mastectomy (ASM). In an analysis of 217 mastectomy specimens the areola was involved in 2 of the 23 cases of positive NACs. This represented $0.9 \%$ of all the mastectomy specimens [81]. Access for ASM is facilitated by medial and lateral extensions to the incision encircling the nipple. This may achieve a superior cosmetic outcome compared to conventional SSM, only requiring a subsequent nipple reconstruction, if requested by the patient. Unfortunately, nipple reconstruction using one of the conventional local flap technique is problematic in this situation.

In a series of $17 \mathrm{ASM}$ and IBRs the only reported complication was a wound infection in one patient. Although 10 of the cases were done for prophylaxis, there was no local or distant recurrence over median follow-up of 24 months [81].

\section{Radiotherapy \& SSM}

Most women undergoing mastectomy for breast cancer 
do not require post-mastectomy radiotherapy (PMRT). However, patients with more than four positive regional lymph nodes and large tumours $(>5 \mathrm{~cm})$ will be offered it in view of the proven reduction in LR and improved survival [82]. There is limited data from a sub-group analysis of Danish trials [83] suggesting there is a survival benefit in patients with $1-3$ positive nodes. The "SUPREMO" trial has been designed to address the benefit in this patient group and in other patients thought to be at intermediate risk of local recurrence [84]. The frequency of PMRT is therefore increasing, which has complicated the planning of IBR as post-reconstruction radiotherapy is associated with local complications [85]. In addition to the potential detrimental effect on cosmesis, the planning and delivery of PMRT can be complicated by the presence of the reconstructed breast itself [86].

The overall complication rate of PMRT following autologous breast reconstruction ranges from $5 \%$ to $16 \%$ [7]. Although results from individual series vary, complications following IBR and PMRT occur in a high proportion of patients [87]. A study of immediate TRAM reconstructions showed the commonest complications were fat necrosis (16\%) and radiation fibrosis (11\%) [88]. Tran et al. have recommended that patients who require PMRT should undergo delayed free TRAM flap reconstruction in order to avoid significant late complications such as fat necrosis, volume loss and flap contracture [89]. Fat necrosis leads to volume loss and hardening of the reconstructed breast and particularly occurs when PMRT is given after IBR using free tissue transfer of skin and fat only (e.g. DIEP flap). For implant or implant-assisted IBR, PMRT can lead to higher rates of significant capsular-contracture resulting in a poor aesthetic outcome. One study compared 39 irradiated implant reconstructed breasts with 338 non-irradiated reconstructions and found a significant negative effect on the reconstructive outcome with implants [90], the main complications being capsular contracture and post-operative pain, $43 \%$ of patients required a capsulotomy. In another retrospective study, 68 IBRs that were irradiated were compared with 75 non-irradiated IBRs. Capsular contracture rates were $68 \%$ vs $40 \%$ respectively, patient satisfaction was $67 \%$ vs $88 \%$. However, $72 \%$ of those irradiated said that they would choose the same form of reconstruction again [91].

A systematic review of capsular contracture revealed that various factors are involved in the development of clinically significant capsules and that although capsulotomy is possible, there is a high recurrence rate [92]. This has led some surgeons to recommend that implantsbased IBR should be avoided if PMRT is likely [87]. Alternative approaches are an autologous IBR or a delayed breast reconstruction. More recently, an "immediate-delayed" reconstructive technique has been suggested.
A temporary tissue expander can be used deep to the pectoralis major muscle at the time of SSM. Following PMRT, delayed reconstruction can then be done, removing the tissue expander and using a myocutaneous flap and/or implant $[7,87]$. This approach could avoid the potential radiotherapy delivery problems and cosmetic disadvantages associated with IBR followed by PMR [87]. Alternatively, prior to mastectomy, the radiological assessment of tumour size combined with analysis of core-biopsies from the primary tumour and techniques such as SNLB could be used to assess the likelihood of PMRT, thereby facilitating the selection of patients for immediate-delayed reconstruction. However, randomised controlled trials are also required to compare immediate-delayed reconstruction to conventional mastectomy and delayed reconstruction for those women who require PMRT.

Pre-operative radiotherapy can also affect the outcome of SSM and IBR. Hultman and Daiza reported the effects of previous radiotherapy on subsequent SSM and IBR in 37 breasts, although not all patients had received previous radiotherapy [93]. TRAMs, LDs and implants were included. 9 patients $(24 \%)$ had a SSM flap complication of which 5 required re-operations. Adjuvant treatment with chemotherapy or radiotherapy was not delayed in these patients. Previous irradiation and diabetes were found to be significant risk factors for complications. Chang et al. found a significantly higher frequency of native flap compromise and capsular contracture in women who had received pre-operative radiotherapy prior to SSM. TRAM flap reconstruction was also found to be superior to LD + implant after SSM in this group of patients [94]. These studies are small and larger studies with longer follow-up are required to verify their findings. However, it would appear that women with previously irradiated breasts can still benefit from the advantages of SSM and IBR using autologous tissue, as long as the risk of skin complications is explained [63].

\section{Conclusion}

Despite their shortcomings, numerous retrospective studies have supported the growing body of evidence that SSM is oncologically adequate for early-stage IBC and DCIS. In some cases it is safe to preserve the NAC, providing the tumour is remote and a frozen-section protocol is followed. Endoscopic approaches to the breast are in their infancy and superiority over conventional techniques has yet to be established. Patients with IBC and a clinically negative axilla should undergo SLNB, either pre- or intra-operatively. SSM can facilitate IBR, providing an aesthetically superior treatment option for women who are not suitable for breast conserving surgery. The optimal cosmetic outcome is found in women who have not had prior RT and those who are unlikely to 
need PMRT. Though neither represents a contraindication to SSM and IBR, the optimal integration of IBR and RT has yet to be determined. Current reconstructive options are reliable and associated with low morbidity and high levels of patient satisfaction. Dual procedures afford many advantages including fewer hospital admissions and a reduced need for contra-lateral breast adjustment in order to achieve symmetry. Good outcomes require appropriate patient selection, a coordinated oncoplastic and multidisciplinary approach. Patients should be appropriately counselled and photographs can be used to illustrate the likely outcome and potential complications.

\section{Search Strategy and Selection Criteria}

Articles were identified by searches of MEDLINE and PubMed up to October 2011 using the terms: "Skin Sparing Mastectomy" or "Subcutaneous Mastectomy" or "Nipple Sparing Mastectomy" or "Areola Sparing mastectomy" or "Endoscopic Mastectomy" and "Breast Cancer" or "DCIS" and "Oncological Safety" or "Aesthetic" or "Cosmesis" and "Morbidity" or "Mortality" and "Sentinel Lymph Node Biopsy" or "Radiotherapy" and "Evidence" and "Recurrence". Studies identified were screened for those that focused on Skin Sparing Mastectomy. All randomized controlled trials and large retrospective series were included. The reference articles in this review were selected to provide a balanced and representative overview of a complex subject with an extensive base of published work.

\section{REFERENCES}

[1] B. Fisher, S. Anderson, J. Bryant, R. G. Margolese, M. Deutsch, E. R. Fisher, J. H. Jeong and N. Wolmark, "Twenty-Year Follow-Up of a Randomized Trial Comparing Total Mastectomy, Lumpectomy, and Lumpectomy plus Irradiation for the Treatment of Invasive Breast Cancer," New England Journal of Medicine, Vol. 347, 2002, pp. 1233-1241. doi:10.1056/NEJMoa022152

[2] NICE Guideline CG80, "Early and Locally Advanced Breast Cancer: Diagnosis and Treatment," 2009. http://guidance.nice.org.uk/CG80/Guidance/pdf/English

[3] D. A. Hidalgo, "Aesthetic Refinement in Breast Reconstruction: Complete Skin-Sparing Mastectomy with Autogenous Tissue Transfer," Plastic \& Reconstructive Surgery, Vol. 102, No. 1, 1998, pp. 63-70. doi:10.1097/00006534-199807000-00009

[4] B. A. Toth and P. Lappert, "Modified Skin Incisions for Mastectomy: The Need for Plastic Surgical Input in PreOperative Planning," Plastic \& Reconstructive Surgery, Vol. 87, No. 6, 1991, pp. 1048-1053. doi:10.1097/00006534-199106000-00006

[5] G. W. Carlson, "Skin Sparing Mastectomy: Anatomic and Technical Considerations," American Surgeon, Vol. 62, No. 2, 1996, pp. 151-155.

[6] R. M. Simmons and T. L. Adamovich, "Skin-Sparing
Mastectomy," Surgical Clinics of North America, Vol. 83, No. 4, 2003, pp. 885-899. doi:10.1016/S0039-6109(03)00035-5

[7] S. E. Singletary, "Skin-Sparing Mastectomy with Immediate Breast Reconstruction: The M. D. Anderson Cancer Center Experience," Annals of Surgical Oncology, Vol. 3, No. 4, 1996, pp. 411-416. doi:10.1007/BF02305673

[8] G. W. Carlson, A. Losken, B. Moore, J. Thornton, M. Elliott, G. Bolitho and D. D. Denson, "Results of Immediate Breast Reconstruction after Skin-Sparing Mastectomy," Annals of Plastic Surgery, Vol. 46, No. 3, 2001, pp. 222-228. doi:10.1097/00000637-200103000-00003

[9] J. Shen, J. Ellenhorn, D. Qian, D. Kulber and J. Aronowitz, "Skin-Sparing Mastectomy: A Survey Based Approach to Defining Standard of Care," American Surgeon, Vol. 74, No. 10, 2008, pp. 902-905.

[10] R. K. Gendy, J. A. Able and R. M. Rainsbury, "Impact of Skin-Sparing Mastectomy with Immediate Reconstruction and Breast-Sparing Reconstruction with Miniflaps on the Outcomes of Oncoplastic Breast Surgery," British Journal of Surgery, Vol. 90, No. 4, 2003, pp. 433-439. doi:10.1002/bjs.4060

[11] S. Shrotria, "The Peri-Areolar Incision-Gateway to the Breast!" European Journal of Surgical Oncology, Vol. 27, No. 6, 2001, pp. 601-603. doi:10.1053/ejso.2001.1051

[12] D. C. Hammond, P. A. Capraro, E. B. Ozolins and J. F. Arnold, "Use of a Skin-Sparing Reduction Pattern to Create a Combination Skin-Muscle Flap Pocket in Immediate Breast Reconstruction," Plastic \& Reconstructive Surgery, Vol. 110, No. 1, 2002, pp. 206-211. doi:10.1097/00006534-200207000-00035

[13] S. E. Singletary and G. L. Robb, "Oncologic Safety of Skin-Sparing Mastectomy," Annals of Surgical Oncology, Vol. 10, No. 2, 2003, pp. 95-97. doi:10.1245/ASO.2003.01.910

[14] G. W. Carlson, J. Bostwick III, T. N. Styblo, B. Moore, J. T. Bried, D. R. Murray and W. C. Wood, "Skin Sparing Mastectomy, Oncologic and Reconstructive Considerations," Annals of Surgery, Vol. 225, No. 5, 1997, pp. 570575. doi:10.1097/00000658-199705000-00013

[15] B. L. Stradling, M. Ahn, J. Angelats and S. G. Gabram, "Skin-Sparing Mastectomy with Sentinel Lymph Node Dissection: Less Is More," Archives Surgery, Vol. 136, No. 9, 2001, pp. 1069-1075. doi:10.1001/archsurg.136.9.1069

[16] T. J. Meretoja, T. A. Jahkola, T. S. Toivonen, L. A. Krogerus, P. S. Heikkila, K. A. von Smitten and M. H. Leidenius, "Sentinel Node Biopsy with Intraoperative Diagnosis in Patients Undergoing Skin-Sparing Mastectomy and Immediate Breast Reconstruction," European Journal of Surgical Oncology, Vol. 33, No. 10, 2007, pp. 1146-1149.

[17] H. M. Kuerer, S. Krishnamurthy and S. J. Kronowitz, "Important Technical Considerations for Skin-Sparing Mastectomy with Sentinel Lymph Node Dissection," Archives of Surgery, Vol. 137, No. 6, 2002, p. 747. doi:10.1001/archsurg.137.6.747-a

[18] P. Schrenk, S. Woelfl, S. Bogner, F. Moser and W. Wayand, "The Use of Sentinel Node Biopsy in Breast 
Cancer Patients Undergoing Skin Sparing Mastectomy and Immediate Autologous Reconstruction," Plastic \& Reconstructive Surgery, Vol. 116, No. 5, 2005, pp. 12781286. doi:10.1097/01.prs.0000181515.11529.9a

[19] L. D. Friedlander, J. Sundin and N. Bakshandeh, "Endoscopy Mastectomy and Breast Reconstruction: Endoscopic Breast Surgery," Aesthetic Plastic Surgery, Vol. 19, No. 1, 1995, pp. 27-29. doi:10.1007/BF00209307

[20] K. Yamashita and K. Shimizu, "Endoscopic Video-Assisted Breast Surgery: Procedures and Short-Term Results," Journal of Nippon Medical School, Vol. 73, No. 4, 2006, pp. 193-202. doi:10.1272/jnms.73.193

[21] E. K. Lee, S. H. Kook, Y. L. Park and W. G. Bae, "Endoscopy-Assisted Breast-Conserving Surgery for Early Breast Cancer," World Journal of Surgery, Vol. 30, No. 6, 2006, pp. 957-964. doi:10.1007/s00268-005-0202-y

[22] Y. Tamaki, Y. Tsukamoto, Y. Miyoushi, T. Taguchi and S. Noguchi, "Overview: Video Assisted Breast Surgery," Biomedicine \& Pharmacotherapy, Vol. 56, Suppl. 1, 2002, pp. 187-191. doi:10.1016/S0753-3322(02)00206-8

[23] H. Nakajima, K. Sakaguchi, N. Mizuta, T. Hachimine, S. Ohe and K. Sawai, "Video-Assisted Total Glandectomy and Immediate Reconstruction for Breast Cancer," Biomedicine \& Pharmacotherapy, Vol. 56, Suppl. 1, 2002, pp. 205-208.

[24] W. S. Ho, S. Y. Ying and A. C. Chan, "Endoscopic-Assisted Subcutaneous Mastectomy and Axillary Dissection with Immediate Mammary Prosthesis Reconstruction for Early Breast Cancer," Surgical Endoscopy and Other Interventional Techniques, Vol. 16, No. 2, 2002, pp. 302306. doi:10.1007/s004640000203

[25] K. Kitamura, M. Ishida, H. Inoue, J. Kinoshita, M. Hashizume and K. Sugimachi, "Early Results of an EndoscopeAssisted Subcutaneous Mastectomy and Reconstruction for Breast Cancer," Surgery, Vol. 131, Suppl. 1, 2002, pp. 324-329. doi: 10.1067/msy.2002.120120

[26] S. S. Kroll, "Immediate Breast Reconstruction. A Review," Annales Chirurgiae et Gynaecologiae, Vol. 86, No. 1, 1997, pp. 5-12.

[27] J. W. Granzow, J. L. Levine, E. S. Chiu and R. J. Allen, "Breast Reconstruction Using Perforator Flaps," Journal of Surgical Oncology, Vol. 94, No. 6, 2006, pp. 441-454. doi:10.1002/jso.20481

[28] M Salgarello, A. Seccia and F. Eugenio, "Immediate Breast Reconstruction with Anatomical Permanent Expandable Implants after Skin-Sparing Mastectomy: Aesthetic and Technical Refinements," Annals of Plastic Surgery, Vol. 52, No. 4, 2004, pp. 358-364. doi:10.1097/01.sap.0000105914.69611.c7

[29] L. A. Woerdeman, J. J. Hage, M. M. Hofland and E. J. Rutgers, "A Prospective Assessment of Surgical Risk Factors in 400 Cases of Skin-Sparing Mastectomy and Immediate Breast Reconstruction with Implants to Establish Selection Criteria," Plastic and Reconstructive Surgery, Vol. 119, No. 2, 2007, pp. 455-463. doi:10.1097/01.prs.0000246379.99318.74

[30] L. A. Woerdeman, J. J. Hage, M. J. Smeulders, E. J. Rutgers and C. M. van der Horst, "Skin-Sparing Mastectomy and Immediate Breast Reconstruction by Use of Implants:
An Assessment of Risk Factors for Complications and Cancer Control in 120 Patients," Plastic and Reconstructive Surgery, Vol. 118, No. 2, 2006, pp. 321-330. doi:10.1097/01.prs.0000234049.91710.ba

[31] H. Sbitany, A. N. Amalfi and H. N. Langstein, "Preferences in Choosing between Breast Reconstruction Options: A Survey of Female Plastic Surgeons," Plastic and Reconstructive Surgery, Vol. 124, No. 6, 2009, pp. 17811789. doi:10.1097/PRS.0b013e3181bf8056

[32] M. Y. Nahabedian, "Postmastectomy Reconstruction: An Approach to Patient Selection," Plastic and Reconstructive Surgery, Vol. 124, No. 1, 2009, pp. 43-52. doi:10.1097/PRS.0b013e31818b9005

[33] W. J. Schneider, H. L. Hill and R. G. Brown, "Latissimus Dorsi Myocutaneous Flap for Breast Reconstruction," British Journal of Plastic Surgery, Vol. 30, No. 4, 1977, pp. 277-281. doi:10.1016/0007-1226(77)90117-5

[34] J. I. de la Torre, R. J. Fix, P. M. Gardner and L. O. Vasconez, "Reconstruction with the Latissimus Dorsi Flap after Skin-Sparing Mastectomy," Annals of Plastic Surgery, Vol. 46, No. 3, 2001, pp. 229-233. doi:10.1097/00000637-200103000-00004

[35] C. R. Hartrampf, M. Scheflan and P. W. Black, "Breast Reconstruction with a Transverse Abdominal Island Flap," Plastic and Reconstructive Surgery, Vol. 69, No. 2, 1982, pp. 216-225. doi:10.1097/00006534-198202000-00006

[36] Z. M. Arnez, J. Bajec, A. F. Bardsley, T. Scamp and M. H. Webster, "Experience with 50 Free TRAM Flap Breast Reconstructions," Plastic and Reconstructive Surgery, Vol. 87, No. 3, 1991, pp. 470-478. doi:10.1097/00006534-199103000-00014

[37] M. Drucker-Zertuche and C. Robles-Vidal, "A 7-Year Experience with Immediate Breast Reconstruction after Skin Sparing Mastectomy for Cancer," European Journal of Surgical Oncology, Vol. 33, No. 2, 2007, pp. 140-146. doi:10.1016/j.ejso.2006.10.010

[38] G. Gherardini, R. Thomas, G. Basoccu, R. Zaccheddu, L. Fortunato, P. Cortino, G. R. Evans, A. Matarasso, M. D'Aiuto and G. D'Aiuto, "Immediate Breast Reconstruction with the Transverse Rectus Abdominis Musculocutaneous Flap after Skin-Sparing Mastectomy," International Surgery, Vol. 86, No. 4, 2001, pp. 246-251.

[39] H. Holmstrom, "The Free Abdominoplasty Flap and Its Use in Breast Reconstruction," Scandinavian Journal of Plastic and Reconstructive Surgery and Hand Surgery, Vol. 13, No. 3, 1979, pp. 423-427. doi:10.3109/02844317909013092

[40] P. N. Blondeel, "The Sensate Free Superior Gluteal Artery Perforator (S-GAP) Flap: A Valuable Alternative in Autologous Breast Reconstruction," British Journal of Plastic Surgery, Vol. 52, No. 3, 1999, pp. 185-193. doi:10.1054/bjps.1998.3032

[41] K. Yano, K. Hosokawa, K. Nakai, T. Kubo, R. Hattori, T. Taguchi, Y. Tamaki and S. Noguchi, "Skin-Sparing Mastectomy and Immediate Reconstruction with a Deep Inferior Epigastric Perforator Flap," Breast Cancer, Vol. 10, No. 3, 2003, pp. 275-280. doi:10.1007/BF02967663

[42] A. M. Munhoz, E. Arruda, E. Montag, C. Aldrighi, J. M. 
Aldrighi, R. Gemperli and M. C. Ferreira, "Immediate Skin-Sparing Mastectomy Reconstruction with Deep Inferior Epigastric Perforator (DIEP) Flap. Technical Aspects and Outcome," Breast Journal, Vol. 13. No. 5, 2007, pp. 470-478. doi:10.1111/j.1524-4741.2007.00467.x

[43] V. F. Cocquyt, P. N. Blondeel, H. T. Depypere, K. A. Van De Sijpe, K. K. Daems, S. J. Monstrey and S. J. Van Belle, "Better Cosmetic Results and Comparable Quality of Life after Skin-Sparing Mastectomy and Immediate Autologous Breast Reconstruction Compared to Breast Conservative Treatment," British Journal of Plastic Surgery, Vol. 56, No. 5, 2003, pp. 462-470. doi:10.1016/S0007-1226(03)00198-X

[44] T. Fujino, T. Harasina and F. Aoyagi, "Reconstruction for Aplasia of the Breast and Pectoral Region by Microvascular Transfer of a Free Flap from the Buttock," Plastic and Reconstructive Surgery, Vol. 56, No. 2, 1975, pp. 178-181. doi:10.1097/00006534-197508000-00010

[45] R. J. Allen and C. Tucker Jr., "Superior Gluteal Artery Perforator Free Flap for Breast Reconstruction," Plastic and Reconstructive Surgery, Vol. 95, No. 7, 1995, pp. 1207-1212. doi:10.1097/00006534-199506000-00010

[46] A. B. Guerra, S. E. Metzinger, R. S. Bidros, P. S. Gill, C. L. Dupin and R. J. Allen, "Breast Reconstruction with Gluteal Artery Perforator (GAP) Flaps: A Critical Analysis of 142 Cases," Annals of Plastic Surgery, Vol. 52, No. 2, 2004, pp. 118-125. doi:10.1097/01.sap.0000095437.43805.d1

[47] Z. M. Arnez, D. Pogorelec, F. Planinsek and U. Ahcan, "Breast Reconstruction by the Free Transverse Gracilis (TUG) Flap," British Journal of Plastic Surgery, Vol. 57, No. 1, 2004, pp. 20-26. doi:10.1016/j.bjps.2003.10.007

[48] G. Wechselberger and T. Schoeller, "The Transverse Myocutaneous Gracilis Free Flap: A Valuable Tissue Source in Autologous Breast Reconstruction," Plastic and Reconstructive Surgery, Vol. 114, No. 1, 2004, pp. 69-73. doi:10.1097/01.PRS.0000127797.62020.D4

[49] A. G. Jimenez, P. St. Germain, M. Sirois, M. Hatheway and R. Lethbridge, "Free Omental Flap for Skin-Sparing Breast Reconstruction Harvested Laparoscopically," Plastic and Reconstructive Surgery, Vol. 110, No. 2, 2002, pp. 545-551. doi:10.1097/00006534-200208000-00028

[50] F. E. Barton Jr., J. M. English, W. B. Kingsley and M. Fietz, "Glandular Excision in Total Glandular Mastectomy and Modified Radical Mastectomy: A Comparison," Plastic and Reconstructive Surgery, Vol. 88, No. 3, 1991, pp. 389-392. doi:10.1097/00006534-199109000-00001

[51] R. Z. Torresan, C. C. dos Santos, H. Okamura and M. Alvarenga, "Evaluation of Residual Glandular Tissue after Skin-Sparing Mastectomies," Annals of Surgical Oncology, Vol. 12, No. 12, 2005, pp. 1037-1044. doi:10.1245/ASO.2005.11.027

[52] C. M. Ho, C. K. Mak, Y. Lau, W. Y. Cheung, M. C. Chan and W. K. Hung, "Skin Involvement in Invasive Breast Carcinoma: Safety of Skin-Sparing Mastectomy," Annals of Surgical Oncology, Vol. 10, No. 2, 2003, pp. 102-107. doi:10.1245/ASO.2003.05.001

[53] L. A. Newman, H. M. Kuerer, K. K. Hunt, S. S. Kroll, F. C. Ames, M. I. Ross, B. W. Feig and S. E. Singletary,
"Presentation, Treatment, and Outcome of Local Recurrence after Skin-Sparing Mastectomy and Immediate Breast Reconstruction," Annals of Surgical Oncology, Vol. 5, No. 7, 1998, pp. 620-626. doi:10.1007/BF02303832

[54] S. Lanitis, P. P. Tekkis, G. Sgourakis, N. Dimopoulos, R. Al Mufti and D. J. Hadjiminas, "Comparison of SkinSparing Mastectomy versus Non-Skin-Sparing Mastectomy for Breast Cancer: A Meta-Analysis of Observational Studies," Annals of Surgery, Vol. 251, No. 4, 2010, pp. 632-639. doi:10.1097/SLA.0b013e3181d35bf8

[55] M. Yi, S. J. Kronowitz, F. Meric-Bernstam, B. W. Feig, W. F. Symmans, A. Lucci, M. I. Ross, G. V. Babiera, H. M. Kuerer and K. K. Hunt, "Local, Regional, and Systemic Recurrence Rates in Patients Undergoing SkinSparing Mastectomy Compared with Conventional Mastectomy," Cancer, Vol. 117, No. 5, 2011, pp. 916-924. doi: $10.1002 /$ cncr.25505

[56] K. J. Downes, B. S. Glatt, S. K. Kanchwala, R. Mick, D. L. Fraker, K. R. Fox, L. J. Solin, L. P. Bucky and B. J. Czerniecki, "Skin-Sparing Mastectomy and Immediate Reconstruction Is an Acceptable Treatment Option for Patients with High-Risk Breast Carcinoma," Cancer, Vol. 103, No. 5, 2005, pp. 906-913. doi:10.1002/cncr.20851

[57] R. D. Foster, L. J. Esserman, J. P. Anthony, E. S. Hwang and H. Do, "Skin-Sparing Mastectomy and Immediate Breast Reconstruction: A Prospective Cohort Study for the Treatment of Advanced Stages of Breast Carcinoma,' Annals of Surgical Oncology, Vol. 9, No. 5, 2002, pp. 462-466. doi:10.1007/BF02557269

[58] T. M. Allweis, M. E. Boisvert, S. E. Otero, et al., "Immediate Reconstruction after Mastectomy for Breast Cancer Does Not Prolong the Time to Starting Adjuvant Chemotherapy," American Journal of Surgery, Vol. 183, 2002, 218-21. doi:10.1016/S0002-9610(02)00793-6

[59] R. M. Rainsbury, "Skin-Sparing Mastectomy," British Journal of Surgery, Vol. 93, No. 3, 2006, pp. 276-281. doi:10.1002/bjs.5257

[60] H. N. Langstein, M. H. Cheng, S. E. Singletary, G. L. Robb, E. Hoy, T. L. Smith and S. S. Kroll, "Breast Cancer Recurrence after Immediate Reconstruction: Patterns and Significances," Plastic and Reconstructive Surgery, Vol. 111, No. 2, 2003, pp. 712-720. doi:10.1097/01.PRS.0000041441.42563.95

[61] G. W. Carlson, A. Page, E. Johnson, K. Nicholson, T. M. Styblo and W. C. Wood, "Local Recurrence of Ductal Carcinoma in Situ after Skin-Sparing Mastectomy," Journal of the American College of Surgeons, Vol. 204, No. 5, 2007, pp. 1074-1078. doi:10.1016/j.jamcollsurg.2007.01.063

[62] K. Mokbel, "Towards Optimal Management of Ductal Carcinoma in Situ of the Breast," European Journal of Surgical Oncology, Vol. 29, No. 2, 2003, pp. 191-197. doi:10.1053/ejso.2002.1425

[63] R. Mokbel and K. Mokbel, "Skin-Sparing Mastectomy and Radiotherapy: An Update," International Seminars in Surgical Oncology, Vol. 17, 2006, pp. 3-35.

[64] I. T. Rubio, N. Mirza, A. A. Sahin, G. Whitman, S. S. Kroll, F. C. Ames and S. E. Singletary, "Role of Speci- 
men Radiography in Patients Treated with Skin-Sparing Mastectomy for Ductal Carcinoma in Situ of the Breast," Annals of Surgical Oncology, Vol. 7, No. 7, 2000, pp. 544-548. doi:10.1007/s10434-000-0544-5

[65] A. J. Spiegel and C. E. Butler, "Recurrence Following Treatment of Ductal Carcinoma in Situ with Skin-Sparing Mastectomy and Immediate Breast Reconstruction," Plastic and Reconstructive Surgery, Vol. 111, No. 2, 2003, pp. 706-711. doi:10.1097/01.PRS.0000041440.12442.05

[66] C. Laronga, B. Kemp, D. Johnston, G. L. Robb and S. E. Singletary, "The Incidence of Occult Nipple-Areola Complex Involvement in Breast Cancer Patients Receiving a Skin-Sparing Mastectomy," Annals of Surgical Oncology, Vol. 6, No. 6, 1999, pp. 609-613. doi:10.1007/s10434-999-0609-z

[67] J. S. Vaidya, "Prediction of Nipple and Areola Involvement in Breast Cancer," European Journal of Surgical Oncology, Vol. 24, No. 1, 1998, pp. 15-16. doi:10.1016/S0748-7983(98)80117-0

[68] R. M. Simmons, M. Brennan, P. Christos, V. King and M. Osborne, "Analysis of Nipple/Areolar Involvement with Mastectomy: Can the Areola Be Preserved?" Annals of Surgical Oncology, Vol. 9, No. 2, 2002, pp. 165-168. doi:10.1007/BF02557369

[69] B. Gerber, A. Krause, T. Reimer, H. Muller, I. Kuchenmeister, J. Makovitzky, G. Kundt and K. Friese, "SkinSparing Mastectomy with Conservation of the NippleAreola Complex and Autologous Reconstruction Is an Oncologically Safe Procedure," Annals of Surgery, Vol. 238, No. 1, 2003, pp. 120-127. doi:10.1097/00000658-200307000-00016

[70] A. Banerjee, S. Gupta and N. Bhattacharya, "Preservation of Nipple-Areola Complex in Breast Cancer-A Clinicopathological Assessment," Journal of Plastic, Reconstructive \& Aesthetic Surgery, Vol. 61, No. 10, 2007, pp. 1195-1198.

[71] A. K. Schecter, M. B. Freeman, D. Giri, E. Sabo and J. Weinzweig, "Applicability of the Nipple-Areola Complex-Sparing Mastectomy: A Prediction Model Using Mammography to Estimate Risk of Nipple-Areola Complex Involvement in Breast Cancer Patients," Annals of Plastic Surgery, Vol. 56, No. 5, 2006, pp. 498-504. doi:10.1097/01.sap.0000216946.83252.e4

[72] Z. Vlajcic, R. Zic, S. Stanec, S. Lambasa, M. Petrovecki and Z. Stanec, "Nipple-Areola Complex Preservation: Predictive Factors of Neoplastic Nipple-Areola Complex Invasion," Annals of Plastic Surgery, Vol. 55, No. 3, 2005, pp. 240-244. doi:10.1097/01.sap.0000171680.49971.85

[73] C. M. Chen, J. J. Disa, V. Sacchini, A. L. Pusic, B. J. Mehrara, C. A. Garcia-Etienne and P. G. Cordeiro, "Nipple-Sparing Mastectomy and Immediate Tissue Expander/ Implant Breast Reconstruction," Plastic and Reconstructive Surgery, Vol. 124, No. 6, 2009, pp. 1772-1780. doi:10.1097/PRS.0b013e3181bd05fd

[74] C. N. Verheyden, "Nipple-Sparing Total Mastectomy of Large Breasts: The Role of Tissue Expansion," Plastic and Reconstructive Surgery, Vol. 101, No. 6, 1998, pp. 1494-1500. doi:10.1097/00006534-199805000-00010
[75] A. L. Komorowski, V. Zanini, L. Regolo, A. Carolei, W. M. Wysocki and A. Costa, "Necrotic Complications after Nipple- and Areola-Sparing Mastectomy," World Journal of Surgery, Vol. 30, No. 8, 2006, pp. 1410-1413. doi:10.1007/s00268-005-0650-4

[76] J. P. Crowe Jr., J. A. Kim, R. Yetman, J. Banbury, R. J. Patrick and D. Baynes, "Nipple-Sparing Mastectomy: Technique and Results of 54 Procedures," Archives of Surgery, Vol. 139, No. 2, 2004, pp. 148-150.

doi:10.1001/archsurg.139.2.148

[77] P. Mustonen, J. Lepistö, A. Papp, M. Berg, T. Pietiläinen, V. Kataja and M. Härmä, "The Surgical and Oncological Safety of Immediate Breast Reconstruction," European Journal of Surgical Oncology, Vol. 30, No. 8, 2004, pp. 817-823. doi:10.1016/S0748-7983(04)00143-X

[78] L. Regolo, B. Ballardini, E. Gallarotti, E. Scoccia and V. Zanini, "Nipple Sparing Mastectomy: An Innovative Skin Incision for an Alternative Approach," Breast, Vol. 17, No. 1, 2007, pp. 8-11.

[79] J. Y. Petit, U. Veronesi, R. Orecchia, A. Luini, P. Rey, M. Intra, F. Didier, S. Martella, M. Rietjens, C. Garusi, F. DeLorenzi, G. Gatti, M. E. Leon and C. Casadio, "Nipple-Sparing Mastectomy in Association with Intra Operative Radiotherapy (ELIOT): A New Type of Mastectomy for Breast Cancer Treatment," Breast Cancer Research and Treatment, Vol. 96, No. 1, 2006, pp. 47-51. doi:10.1007/s10549-005-9033-7

[80] G. Bistoni, A. Rulli, L. Izzo, G. Noya, C. Alfano and F. Barberini, "Nipple-Sparing Mastectomy. Preliminary Results," Journal of Experimental \& Clinical Cancer Research, Vol. 25, No. 4, 2006, pp. 495-497.

[81] R. M. Simmons, S. T. Hollenbeck and G. S. Latrenta, "Two-Year Follow-Up of Areola-Sparing Mastectomy with Immediate Reconstruction," American Journal of Surgery, Vol. 188, No. 4, 2004, pp. 403-406. doi:10.1016/j.amjsurg.2004.07.001

[82] A. Recht and S. B. Edge, "Evidence-Based Indications for Postmastectomy Irradiation," Surgical Clinics of North America, Vol. 83, No. 4, 2003, pp. 995-1013. doi:10.1016/S0039-6109(03)00033-1

[83] M. Overgaard, H. M. Nielsen and J. Overgaard, "Is the Benefit of Postmastectomy Irradiation Limited to Patients with Four or More Positive Nodes, as Recommended in International Consensus Reports? A Subgroup Analysis of the DBCG 82 b \& c Randomized Trials," Radiotherapy \& Oncology, Vol. 82, No. 3, 2007, pp. 247-253. doi:10.1016/i.radonc.2007.02.001

[84] I. H. Kunkler, P. Canney, G. van Tienhoven and N. S. Russell, "Elucidating the Role of Chest Wall Irradiation in 'Intermediate-Risk' Breast Cancer: The MRC/EORTC SUPREMO Trial," Clinical Oncology, Vol. 20, No. 1, 2008, pp. 31-34. doi:10.1016/j.clon.2007.10.004

[85] M. Hussien, B. Salah, A. Malyon and E. M. WielerMithoff, "The Effect of Radiotherapy on the Use of Immediate Breast Reconstruction," European Journal of Surgical Oncology, Vol. 30, No. 5, 2004, pp. 490-494. doi:10.1016/j.ejso.2004.03.005

[86] T. A. Buchholz, S. J. Kronowitz and H. M. Kuerer, "Immediate Breast Reconstruction after Skin-Sparing Mas- 
tectomy for the Treatment of Advanced Breast Cancer: Radiation Oncology Considerations," Annals of Surgical Oncology, Vol. 9, No. 8, 2002, pp. 820-821. doi:10.1007/BF02574506

[87] S. J. Kronowitz and G. L. Robb, "Breast Reconstruction with Postmastectomy Radiation Therapy: Current Issues," Plastic and Reconstructive Surgery, Vol. 114, No. 4, 2004, pp. 950-960. doi:10.1097/01.PRS.0000133200.99826.7F

[88] K. K. Hunt, B. J. Baldwin, E. A. Strom, F. C. Ames, M. D. McNeese, S. S. Kroll and S. E. Singletary, "Feasibility of Postmastectomy Radiation Therapy after TRAM Flap Breast Reconstruction," Annals of Surgical Oncology, Vol. 4, No. 5, 1997, pp. 377-384. doi:10.1007/BF02305549

[89] N. V. Tran, G. R. Evans, S. S. Kroll, B. J. Baldwin, M. J. Miller, G. P. Reece and G. L. Robb, "Postoperative Adjuvant Irradiation: Effects on Tranverse Rectus Abdominis Muscle Flap Breast Reconstruction," Plastic and Reconstructive Surgery, Vol. 106, No. 2, 2000, pp. 313317. doi:10.1097/00006534-200008000-00011

[90] G. R. Evans, M. A. Schusterman and S. S. Kroll, "Reconstruction and the Radiated Breast: Is There a Role for Implants?" Plastic and Reconstructive Surgery, Vol. 96, No. 5, 1995, pp. 1111-1115. doi:10.1097/00006534-199510000-00016
[91] P. G. Cordeiro, A. L. Pusic, J. J. Disa, B. McCormick and K. VanZee, "Irradiation after Immediate Tissue Expander/Implant Breast Reconstruction: Outcomes, Complications, Aesthetic Results, and Satisfaction among $156 \mathrm{~Pa}-$ tients," Plastic and Reconstructive Surgery, Vol. 113, No. 3, 2004, pp. 877-881. doi:10.1097/01.PRS.0000105689.84930.E5

[92] A. Araco, R. Caruso, F. Araco, J. Overton and G. Gravante, "Capsular Contractures: A Systematic Review," Plastic and Reconstructive Surgery, Vol. 124, No. 6, 2009, pp. 1808-1819. doi:10.1097/PRS.0b013e3181bf7f26

[93] C. S. Hultman and S. Daiza, "Skin-Sparing Mastectomy Flap Complications after Breast Reconstruction: Review of Incidence, Management, and Outcome," Annals of Plastic Surgery, Vol. 50, No. 3, 2003, pp. 249-255. doi:10.1097/01.SAP.0000046784.70583.E1

[94] E. I. Chang, D. P. Ly and P. D. Wey, "Comparison of Aesthetic Breast Reconstruction after Skin-Sparing or Conventional Mastectomy in Patients Receiving Preoperative Radiation Therapy," Annals of Plastic Surgery, Vol. 59, No. 1, 2007, pp. 78-81. doi:10.1097/01.sap.0000252487.27077.d6 